\title{
Pathogenetic mechanisms of statin dysglycemia and risk of new-onset diabetes
}

\author{
Maria Pappa', Vasilios Tsimihodimos ${ }^{2}$, Antonios Alaveras ${ }^{3}$ \\ 'First Department of Internal Medicine Konstantopoulio General Hospital N. Ionias-Patision, Athens, Greece \\ ${ }^{2}$ Department of Internal Medicine, School of Medicine, University of loannina, loannina, Greece \\ ${ }^{3}$ Former director of First Department of Internal Medicine General Hospital of Athens Red Cross/Korgialenio-Benakio
}

\begin{abstract}
Statins are the cornerstone for primary and secondary prevention of cardiovascular disease as they have been proven to reduce cardiovascular morbidity and mortality. Statins act by inhibiting 3-hydroxy-3-methylglutarylCoA reductase, the rate limiting enzyme of cholesterol synthesis. Despite their important role in prevention of cardiovascular disease, there is evidence suggesting that they worsen glycaemia increasing the risk of type 2 diabetes by $10-12 \%$, an effect that appears to differ by dose and type of statin used, with higher doses related to higher risk. Statins may cause hyperglycaemia through many pathways e.g., by increasing the intracellular concentration of calcium in the $\beta$-cell, leading to a decrease in insulin release and in addition may decrease the GLUT-4 transporter hindering the peripheral uptake of glucose. Diabetes is a major risk factor for cardiovascular disease, with statins offering remarkable protection, a property which should be stressed to the patient and perhaps even to healthcare personnel when hesitant to prescribe statins.
\end{abstract}

KEY WORDS: Statins, cardiovascular protection, dysglycemiic properties-new-oncet diabetes

\section{INTRODUCTION}

Atherosclerotic cardiovascular disease continues to be a major cause of morbidity and mortality globally. ${ }^{1}$ Major risk factors involved in the pathogenesis of atherosclerosis are hypertension, diabetes, dyslipidaemia, obesity, smoking, a sedentary lifestyle, hemodynamic forces, sex hormonal imbalance (e.g., oestrogen deficiency, menopause) and ageing. Although most risk factors are well known, the pathophysiology of atherosclerotic disease

\section{Corresponding author:}

Vasilios Tsimihodimos

Department of Internal Medicine, School of Medicine,

University of loannina, 45110 loannina, Greece

Tel.: +30 6945770624

E-mail: tsimiho@gmail.com is much more complicated. Our knowledge concerning the endothelium has increase dramatically during the last decades. However, much has yet to be learnt about this vital'organ,' an important integral factor of the arteriosclerotic process. In health, this vascular layer supports the normal functioning of various tissues and organs, whereas its dysfunction in disease is an important contributor to the local and systemic manifestations of atherosclerotic cardiovascular disease.

\section{THE STATINS AND ATHEROSCLEROSIS}

The modified LDLs, and oxidized lipid moieties derived from them, induce endothelial and smooth muscle cells to express adhesion molecules e.g. vascular cell adhesion

Submission: 24.03.2021, Acceptance: 03.05.2021 
molecule - 1 and intercellular adhesion molecule-1, chemoattractants e.g. monocyte chemoattractant protein-1, and growth factors e.g. macrophage colony-stimulating factor and granulocyte-macrophage colony-stimulating factor which interact with receptors on monocytes stimulating their homing, migration, and differentiation into macrophages and dendritic cells. In 1976, the Japanese microbiologist Akira Endo discovered the first statin as a product of the fungus Penicillium citrinum that inhibited the activity of 3 - hydroxy-3- metylglutaryl coenzyme A reductase (HMGCR), which was named compactin. Subsequently, researchers discovered another HMGCR inhibitor derived from Aspergillus terrus, originally named mevinolin and later Lovastatin. Statins block the rate-limiting step of cholesterol synthesis in which HMGCR is transformed to mevalonate by the enzyme HMGCR. However, the cholesterol biosynthesis pathway is also involved in the synthesis of dolichols (required for glycoprotein synthesis), the production of ubiquinone (coenzme Q- which plays an important role in muscle cell energy) and in the process of prenylation of proteins needed in controlling signalling pathways. Although lowering cholesterol levels is the main property of statin, production of other downstream products of the mevalonate pathway may explain both beneficial and deleterious effects of statins.

\section{THE PROPERTIES OF STATINS}

\section{A. Properties favouring the use of statins for dyslipidemia}

\section{i. Lipid-lowering effects}

Large-scale clinical trials have demonstrated that statins substantially reduce cardiovascular-related morbidity and mortality in patients with and without cardiovascular disease. In a large prospective meta-analysis of data from 90,056 participants in 14 randomised trials, statins safely reduced the 5-year incidence of major coronary events, coronary revascularisation, and stroke by about one fifth per $38.67 \mathrm{mg} / \mathrm{dl}(1 \mathrm{mmol} / \mathrm{L})$ reduction in LDL-C. Remarkably, this was achieved irrespective of the initial lipid profile or other characteristics - age, sex, cholesterol levels, presence of diabetes, hypertension, previous myocardial infarction or other coronary heart disease. ${ }^{3}$ Furthermore, for every $38.67 \mathrm{mg} / \mathrm{dl}$ ( $1 \mathrm{mmol} / \mathrm{L}$ ) of LDL-C reduction, the rate of major vascular events decreased by $22 \%$, the vascular mortality by $14 \%$ and the all-cause mortality by $10 \%$ per year. Lowering LDL -C by $77.34 \mathrm{mg} / \mathrm{dl}$ ( $2 \mathrm{mmol} / \mathrm{L}$ for 5 years) in 10,000 patients would reduce the rate of major vascular events by $10 \%$ in secondary prevention (patients at high risk) and by $5 \%$ in primary prevention (patients at lower risk). ${ }^{4}$ In addition to lowering the levels of cholesterol, statins also confer cardiovascular protection by decreasing LDL-C oxidation, promoting the stabilization of the atherosclerotic plaque, inhibiting endothelial dysfunction, vascular smooth muscle proliferation as well as reducing platelet activity.

\section{ii. Pleiotropic effects}

Statins inhibit other downstream products of the mevalonate pathway, generating its pleiotropic effects. By way of these pleiotropic effects, statins modulate virtually all known mechanisms of the atherosclerotic process, and interestingly, with beneficial effects beyond the cardiovascular system. Endothelial dysfunction represents one of the first steps in the pathogenesis of atherosclerosis, entailing, amongst others, impaired nitric oxide (NO) mediated vasodilatation. Statins inhibit the prenylation of Rac and Rho proteins, increasing the expression of endothelium-derived nitric oxide synthetase (eNOS) and nitric oxide production with improvement in potential vasodilatation.

\section{ii. Anti-inflammatory effects}

Statins reduce cardiovascular risk not only by lowering cholesterol, but also by inhibiting inflammation, reducing the production of inflammatory markers e.g. C-reactive protein (CRP), serum amyloid A (SAA), interleukins, and adhesion molecules e.g., intracellular adhesion molecule (ICAM-I) all of which have been associated with the development and recurrence of cardiovascular events. ${ }^{2}$

\section{iv. Immunomodulatory effects}

Evidence suggests that statins exert anti-inflammatory and immunomodulatory activity in autoimmune diseases, including rheumatoid arthritis, ankylosing spondylitis, lupus, vasculitis or systemic sclerosis, among others. ${ }^{5}$ These properties may be modulated through decreased antigen presentation and $\mathrm{T}$ cell activation by restricting expression of the major histocompatibility complex class II (MHC-II) as well as reducing the cell-surface expression of other immunoregulatory molecules e.g. CD3,CD40, CD80. In addition, statins may impair T-lymphocyte, natural-killer cell proliferation and cytotoxicity. Furthermore, statins decrease the expression of cellular adhesion molecules on leukocytes and endothelial cells leading to impaired cell adhesion and migration to areas of inflamation. ${ }^{5}$

\section{v. Anti-thrombotic effect of statins}

Statins may impair blood clot formation by reducing the expression of tissue factor and platelet aggregation, 
diminishing the formation of thrombin and the expression of its receptor on the platelet surface. A decrease in the levels and activity of procoagulant factors, including fibrinogen, and factors V, VII and XIII has been noted, after treatment with statins. Their anti-coagulant properties were demonstrated in the JUPITER study, which noted a decrease in the rate of peripheral venous thromboembolism in patients on rosuvastatin. ${ }^{26}$

\section{vi. Miscellaneous properties of statins}

Use of statin therapy has also been associated in observational studies with lower rates of several other conditions (eg, cancer, infections, chronic obstructive lung disease, and acute respiratory distress syndrome, atrial fibrillation), but those claims have been reliably refuted by randomised trials of adequate size. ${ }^{6}$

\section{B. Properties which do not favour the use of statins}

\section{i. Myotoxicity}

The pathological mechanisms underlying the myotoxicity of statins are not well defined. A direct effect on muscle, or an autoimmune myopathy associated with autoantibodies targeting HMGCR have been implicated. Direct myotoxicity is a rare condition with an estimated accumulated incidence of about 10 - 20 cases per 10,000 statin-treated patients per year. ${ }^{7}$ It is characterized by its resolution after discontinuing treatment, it is dose-dependent, and of variable severity, ranging from isolated muscle pain to severe rhabdomyolysis even leading to renal failure.

\section{ii. Myopathy}

The prevalence of musculoskeletal pain in a metaanalysis from randomized clinical trials is reported to be $0.3 \%$. This is an important point for clinical practice, as it states that myalgia in patients on statin treatment without elevated creatine kinase (CK) levels, is not due to statin therapy. Only 1 case in 10,000 patients treated with statins each year will develop substantial elevations in CK levels and just about 2-3 per 100,000 patients will develop rhabdomyolysis with extremely high CK levels, myoglobinemia, myoglobinuria and acute renal failure. ${ }^{7}$ However, the risk of statin myotoxicity may increase substantially when statins are used in combination with other drugs that affect their metabolism, specially inhibitors of cytochrome P450.

\section{iii. Incidence of diabetes and statins}

Large randomized clinical trials have demonstrated an increased risk of diabetes in patients on statins. The attributable excess risk has been estimated to be about 10-20 per 10,000 patients treated per year, similar to the risk of developing a significant myopathy. ${ }^{8}$ The risk is proportional to the dose used and appears soon after starting therapy. Patients with other risk factors for cardiovascular disease e.g., elevated body mass index, impaired fasting glucose or high $\mathrm{HbA} 1 \mathrm{c}$ are more prone to develop diabetes after taking a statin. ${ }^{8}$ The pathogenesis of statin-related diabetes is unknown. Some of the mechanisms implicated are described in more detail further on. One theory is related to lowering of LDL cholesterol or the increasing number of LDL receptors induced by statins, enabling more cholesterol to enter and damaging the pancreatic cell.

\section{iv. Other side effects of statin therapy}

Although reported to increase serum levels of liver enzymes, statin administration is rarely associated with serious liver injury - about 1 case per 100,000 users. One should always keep in mind that the same enzymes are to be found in the muscle cell, including the myocardium. Determining the levels of creatine kinase (a muscle enzyme) and gamma-glutamyl transferase (a liver enzyme) can help differentiate the aetiology of increased enzyme levels.

\section{v. Miscellaneous side effects}

It remains in question as to whether statins have side effects in cognition. Likewise, there does not seem to be any risk in the incidence of cataract or microvascular retinopathy. Data regarding cancer risk is conflicting. A meta-analysis of randomized clinical trials concluded that statin treatment was not associated with cancer. Reports referring to quality of life, sleep disturbance, suicidal behaviour, neuropathy, and erectile dysfunction have not been proven to be related to statins. ${ }^{6}$

\section{STATINS IN CLINICAL PRACTICE AND THE RISK OF NEW ONSET T2DM}

Statin treatment is the cornerstone of primary and secondary prevention of cardiovascular disease and have been shown to be effective in reducing both LDL-C and cardiovascular disease in diabetic and non-diabetic individuals, are safe and relatively well tolerated. Nevertheless, observational ${ }^{9}$ and clinical studies ${ }^{10}$ as well as meta-analyses ${ }^{8,11,12}$ have found statins to increase the risk of disrupting glucose homeostasis in diabetic as well as non-diabetic individuals. It is estimated that the associated increased risk of diabetes associated with the use of statins is $10-12 \%$. 


\section{A. Observation Studies}

Findings from observational studies have shown an increased risk of T2DM during statin administration in several populations. Despite the significant variability between these studies and the statin administered, the risk ratios (HR) were statistically significant ranging from 1.19 - 1.57, after a follow-up of 3-6 years. ${ }^{4}$ An increased risk of new onset type 2 diabetes was noted in different communities, 22\% in Canada, 15\% in Taiwan and 20\% in Ireland. ${ }^{13,14}$ The Cohort Metabolic Syndrome (METSIM) study, which involved a total of 8,749 non-diabetic men aged 45-73 years, assessed the effect of statin therapy on Fasting Plasma Glucose (FPG), 2-h PG, in relation to an oral glucose tolerance test (OGTT), HbA1c and indices of insulin sensitivity and insulin secretion. (HOMA-IR, HOMA - $B$ and the development of new cases of type 2 diabetes. After a follow-up of 6 years, of those on statin therapy, 625 were diagnosed with new type 2 diabetes - a 46\% increase in the risk of T2DM (HR 1.46 [95\% Cl 1.22, 1.74]), together with an increase in fasting plasma glucose. b) In addition, statins were associated with a $24 \%$ reduction in insulin sensitivity and a $12 \%$ reduction insulin secretion, compared with those not treated with statins. ${ }^{15}$ In a retrospective analysis comparing the effects of atorvastatin $10 \mathrm{mg} /$ day, pravastatin $10 \mathrm{mg} /$ day, and pitavastatin $2 \mathrm{mg} /$ day on glycemic control in patients with T2DM during a 3-month treatment period, only atorvastatin significantly increased FPG and $\mathrm{HbA} 1 \mathrm{c}$. Neither pravastatin nor pitavastatin had any significant influence on FPG and HbA1c. ${ }^{16}$

\section{B. Large, long-term, randomized, double-blind, placebo-controlled studies with statins}

JUPITER, a randomized control trial (RCT), primary prevention intervention study, in which 17,603 men and women without a previous history of cardiovascular disease or T2DM, participated, compared rosuvastatin 20 $\mathrm{mg}$ to placebo. An analysis and evaluation was done for vascular benefits and the risk of diabetes from the use of statins ${ }^{10}$ a) Participants with one or more significant risk factors for diabetes $(n=11508$ ) had a higher risk of developing diabetes than those with no significant risk factor ( $\mathrm{n}$ $=6095)$. The increase in new onset T2DM was 28\% (1 -28, $1 \cdot 07-1 \cdot 54, \mathrm{p}=0.01$ ) among those with risk factors. There was a $39 \%$ reduction in the risk of cardiovascular events and deaths from statins ([HR] 0.61,95\% Cl $0.47-0.79$, $\mathrm{p}=0.0001)$. b) For study participants without major risk factors for diabetes, statins were associated with a $52 \%$ reduction in vascular events and deaths (HR $0.48,95 \% \mathrm{CI}$ $0.33-0.68, p=0.0001)$, and no increase in the incidence of diabetes (0.99-0.45-2.21, $\mathrm{p}=0.99)$. In the 470 individuals who developed T2DM, statin increased the risk by $25 \%$ (HR 1, 25, 95\% Cl 1.05-1.49, p = 0.01) (absolute increase of $0.6 \%)$, while the reduction in cardiovascular events and death was $64 \%$ (HR 1,25, 95\% Cl 1.05-1.49, p = 0.01). Therefore, in this primary prevention study the prospective cardiovascular benefit and mortality from statin therapy outweigh the risk of new diabetes, including participants at high risk for diabetes.

Another RCT study looking at the effectiveness of additional cholesterol and homocysteine reductions, report a dose-dependent increased risk of diabetes in $11.6 \%$ of participants receiving $80 \mathrm{mg}$ of simvastatin compared with $10.9 \%$ receiving $20 \mathrm{mg}$ simvastatin. In a secondary Cox regression analysis, 2,739 patients with T2DM were randomized to receive atorvastatin $10 \mathrm{mg}$ or placebo. Atorvastatin increased $\mathrm{HbA} 1 \mathrm{c}$ by $\geq 0.5 \%$ in $73.6 \%$ of individuals with diabetes in the placebo group, compared to $78.1 \%$ in the atorvastatin group (HR 1.18 [95\% Cl 1.08, 1.29], $\mathrm{P}$ $<0.001)$, a very small but important difference which does not affect the protective effects on cardiovascular disease by atorvastatin. ${ }^{17,18,19}$

Three RCTs with atorvastatin examining the risk of new cases of diabetes were as follows: ${ }^{20} 1$ ) In the TNT study, 239 of the 3,737 patients randomized to atorvastatin 80 $\mathrm{mg} /$ day and 208 of 3,724 to $10 \mathrm{mg}$ developed new onset diabetes (9.24\% vs. $8.11 \%$, [HR]: $1.10,95 \%$ [CI]: 0.94 to 1.29 , $\mathrm{p}=0.226)$. 2). In the IDEAL study, 239 of 3,737 patients randomized to atorvastatin $80 \mathrm{mg} /$ day, and 208 of 3,724 randomised to simvastatin $20 \mathrm{mg} /$ day developed new onset type 2 diabetes (6.40\% vs. 5.59\%, HR: $1.19,95 \% \mathrm{Cl}$ : 0.98 to $1.43, p=0.072$ ). 3) Finally, in the SPARCL study, 166 of 1,905 patients randomized to atorvastatin $80 \mathrm{mg}$ / day and 115 of 1,898 randomised in placebo patients developed new type 2 diabetes (8.71\% vs. 6.06\%, HR: 1.37, 95\% $\mathrm{Cl}$ : 1.08 to $1.75, \mathrm{p} 0.011$ ). In all studies, FPG, hypertension, $\mathrm{BMI}$, triglyceride, and in general all the parameters of the metabolic syndrome, were independent prognostic risk factors for the development of neT2DM. (HR: 1.02, 95\% Cl: 0.77 to $1.35, p=0.69$ ). Adding ezetimibe to a statin was not found to increase the risk of new onset type 2 diabetes. ${ }^{19}$

\section{Meta-Analyses}

In a meta-analysis of 5 RCTs statin trials (PROVE-IT-TIMI, A to Z, TNT, IDEAL, SEARCH), with 32752 participants without a history of diabetes at baseline, 2749 developed new onset type 2 diabetes ( 1449 assigned intensive-dose therapy, 1300 assigned moderate-dose therapy, representing 2.0 additional cases in the intensive-dose group per 1000 patient-years) and 6684 experienced cardiovascular events (3134 and 3550, respectively, representing 6.5 fewer cases in the intensive-dose group per 1000 patient- 
years) over a weighted mean (SD) follow-up of 4.9 (1.9) years). ${ }^{21}$ Odds ratios were 1.12 (95\% confidence interval [Cl], 1.04-1.22; $R^{2}=0 \%$ ) for new-onset diabetes and 0.84 $\left(95 \% \mathrm{Cl}, 0.75-0.94 ; l^{2}=74 \%\right)$ for cardiovascular events for participants receiving intensive therapy compared with moderate-dose therapy. ${ }^{21}$ Conclude from this in a pooled analysis from 5 statin trials, intensive-dose statin therapy was associated with an increased risk of new-onset diabetes compared with moderate-dose statin therapy. It therefore appears that the risk of developing new onset type 2 diabetes from statin is quite small, in contrast to their benefits of cardiovascular risk protection which cannot be compared to the risk of new onset type 2 diabetes. ${ }^{16}$ In a meta-analysis including 20 studies, 18 cohorts and 2 case studies, the overall risk of new onset T2DM was higher in statin users than in those not taking statins (RR 1.44, 95\% Cl 1.31-1.58). Estimates for individual statins showed an effect for rosuvastatin (RR 1.61, 1.30-1.98) and simvastatin (RR 1.38, 1.19-1.61). Atorvastatin and rosuvastatin were associated with a higher risk of new onset type 2 diabetes in patients with the metabolic syndrome..$^{5,8,12,21,22} \mathrm{~A}$ metaanalysis of the Cholesterol Treatment Trialists' (CTT) Collaborators data was analyzed for 18, 686 people with and 71,370 without diabetes in 14 RCTs randomized studies with statin therapy and an average follow-up of 4.3 years. Decreased LDL-C was associated with a $21 \%$ reduction in the incidence of any major vascular event in both those with and without diabetes, directly correlated with an increased risk of developing new onset type 2 diabetes ${ }^{2}$ Statin treatment significantly reduced all-cause mortality (RR) $0.90(\mathrm{Cl}) 0.86-0.94, \mathrm{P} \leq 0.0001$ ) in a large meta-analysis of 76 randomized studies with 170,255 participants, and 14,878 deaths. ${ }^{12}$ Side effects were generally mild, but reported that 17 of the RCTs were associated with an increased risk of new cases of type 2 diabetes (OR 1, 09, $95 \% \mathrm{Cl} 1,02-1,17, \mathrm{P}=0,001)$. In a meta-analysis of 6 studies (WOSCOPS, HPS, LIPID, ASCOT, JUPITER, CORONA) with 57,593 patients on various statins, and a follow-up of 3.4 years, 2,082 patients developed new onset type 2 diabetes. In other words, a small increase in the risk of diabetes (RR 1, 13 [95\% CI 1, 03-1, 23]) was observed without evidence of heterogeneity in all studies. ${ }^{12}$ In a 14year study, 471,250 patients without a history of diabetes, $48.3 \%$ received statins for primary prevention and $51.7 \%$ for secondary prevention. After adjustment, and in comparison to patients on pravastatin, atorvastatin increased the risk of new type 2 diabetes by $22 \%$ (RR 1,22, 95\% Cl1 $15-1,29)$. A significantly increased risk with rosuvastatin by $18 \%(O R 1.18,1,01, .26)$ and simvastatin by $10 \%$ (OR $1.10,1.0 .4-1.17$ ) A meta-analysis of 29 RCTs randomized studies included 163,039 participants, of whom 141,863 non-diabetic. Statins as a class, significantly increased the risk of developing type 2 diabetes by $12 \%$ (grouped OR $1.12,95 \% \mathrm{Cl} 1,05-1,21) \mathrm{p}=0.002$. Atorvastatin $80 \mathrm{mg}$, was associated with a higher risk of type 2 diabetes, with an OR 1.34 (95\% Cl 1, 14-1,57) followed by rosuvastatin (OR: 1, 17, 95\% Cl: 1, 02-1, 35), OR (95\% Cls) and simvastatin $80 \mathrm{mg}$, (1.21 (0.99-1.49). Between rosuvastatin and atorvastatin, rosuvastatin has been associated with the highest risk of T2DM. New onset type 2 diabetes is more common in those with pre-existing risk factors, with an incidence of $14.3 \%$ in the high-dose treated group, and $11.9 \%$ in the moderate dose treated group. ${ }^{32,24}$

\section{Differences in diabetic effects between hydrophilic and lipophilic statins}

As has been referred to, lipophilic statins (atorvastatin, simvastatin, lovastatin, fluvastatin, and pitavastatin) may be more diabetogenic than hydrophilic statins (pravastatin and rosuvastatin) as they can easily penetrate extrahepatic cell membranes e.g. the $\beta$-cell, the adipocytes and skeletal muscle cell. In contrast, hydrophilic statins (e.g. pravastatin) are more specific to hepatocytes and are less likely to enter $\beta$-cells or adipocytes. As has already been stated, the harmful properties of statins have been attributed to dose and activity which in turn are mainly related to their lipophilicity. ${ }^{21,27,44}$

\section{MECHANISM OF INCREASED INCIDENCE OF T2DM WITH STATIN THERAPY}

The impressive increase in our understanding the beneficial mechanisms that statins exert on cardiovascular risk protection have also shed light on their possible adverse properties. Of these, the pathogenetic mechanisms involved in statin-induced disturbances of glucose homeostasis are of exceptional importance. These detrimental effects appear to be multifactorial, reducing insulin secretion from the $\beta$-cell, increasing insulin resistance by disrupting several cellular biosynthetic pathways in insulin-sensitive cells - adipocytes, skeletal muscle cells, and hepatocytes. ${ }^{25,26}$ The statins in general increase gluconeogenesis and therefore hepatic glucose production by regulating the gene expression of key enzymes in the insulin signaling pathway as well as down-regulating the GLUT-4 transporter, responsible for glucose uptake by peripheral cells. ${ }^{22,58}$ Furthermore statins can also cause changes in the circulation of free fatty acids (FFAs), disrupting hormone function e.g. adiponectin and leptin, ${ }^{26}$ and possibly intervening in the epigenetic regulation by specific microRNAs involved in the decreased secretion of insulin. ${ }^{26}$ Some of these complex pathophysiological molecular mechanisms of statin-induced T2DM are described in more detail below. ${ }^{30,47}$ 


\section{A. Statin and the dysregulation of the pancreatic $\beta$-cell function}

As is well known, insulin secretion is initiated with the uptake of glucose by the $\beta$-cell of the pancreas, activating glycolysis in $\beta$-cells, increasing the ATP / ADP ratio which acts as a signal that closes the $\mathrm{K}_{\text {ATP }}$ channels, depolarizes the plasma membrane, with consequent activation of voltage-dependent $\mathrm{Ca}^{2+}$ channels, extracellular $\mathrm{Ca}^{2+}$ entry into the $\beta$-cell and finally exocytosis of Insulin. The maintenance of intracellular $\mathrm{Ca}^{2+}$ homeostasis is strictly regulated to ensure normal insulin secretion and the integrity of $\beta$-cell physiology The ATP sensitivity of the $\mathrm{K}_{\mathrm{ATP}}$ channels is modulated by various factors, including PIP2 and acyl CoAs. In addition, ATP and ADP can act as autocrine activators of purine receptors as they are also found in exocytosed insulin granules. Indeed, inhibition of both P2X and P2Y purine receptors decrease glucose-induced insulin secretion. To date, the relationship between statin-mediated inhibition of cholesterol synthesis and reduced activity of the L2 type $\mathrm{Ca}^{2+}$ channel remains unclear. ${ }^{31}$ However, in in-vitro studies simvastatin directly inhibits L-type $\mathrm{Ca}^{2+}$ channels in rat pancreatic islet $\beta$-cells, reducing activity in the channels, by attenuation of mitochondrial function. ${ }^{33,35}$ Compared to normal control cells, simvastatin-treated cells had a lower insulin content, decreased insulin secretion, and less total insulin induced by low glucose level. ${ }^{34}$ More recently, it has been suggested that statins may reduce membrane potential by inhibiting mitochondrial complex II activity, which causes oxidative stress. Treating $\beta$-cells with atorvastatin and pravastatin inhibits GLUT-2 expression in a concentration-dependent manner, ${ }^{29} \mathrm{n}$ contrast to rosuvastatin and pitavastatin, which have shown a slight increase in GLUT-2 expression. ${ }^{29}$. Coenzyme Q10 plays a critical role in energy metabolism. ${ }^{36}$ Not only is it important for antioxidant and cell membrane stabilization, but is also involved in mitochondrial oxidative phosphorylation. Some statins significantly reduce plasma coenzyme Q10 levels, leading to a reduction in mitochondrial oxidative phosphorylation and reduced ATP production. ${ }^{36}$ In addition, statins can inhibit the mevalonate pathway, thereby inhibiting the production of mevalone metabolites, such as isoprenoid and coenzyme Q10, affecting glucose metabolism and insulin sensitivity. In addition, simvastatin may also indirectly inhibit insulin secretion by reducing GLUT2 expression, thereby reducing glucose uptake. Direct inhibition of the mevalone pathway by statins reduces the intracellular concentration of isoprenoids, the end products of the pathway. Isoprenoids are necessary for the post-translational modification of $\mathrm{G}$ protein, which is important for the exocytosis of insulin.

\section{B. Insulin resistance in statin treatment}

Insulin binding to the insulin receptor (INSR) ${ }^{49}$ activates the pathway aiming at normalizing high blood glucose levels, as well as promoting the conversion of nutrients (glucose, amino acids and fatty acids) into storage macromolecules, such as glycogen, protein and lipids. Binding of insulin to its receptor initiates the auto-phosphorylation of tyrosine residues, the insulin receptor substrate (IRS) ${ }^{49}$, substrates that have just been phosphorylated, triggering several downstream signals ${ }^{50}$ e.g. Akt, PKC, SIK2, mTOR and ROCK $1 .{ }^{50,51}$ IRS-1 activates PI3K, which in turn activates Akt, amongst other targets. Akt activation leads to glucose uptake by facilitating the transfer of GLUT-4 to the plasma membrane ${ }^{53}$ as GLUT-4 is an insulin-dependent transporter. ${ }^{54}$ Akt also promotes glycogen synthesis by inhibiting glycogen synthase 24,25

\section{i. Fatty tissue and statins}

Statin therapy is now known to disrupt the transmission of insulin signal to adipocytes, including INSR, GLUT-4, Akt, and certain small GTP (G-protein) binding proteins. Numerous studies have shown that atorvastatin and lovastatin reduce expression of GLUT-4 in plasma membrane in $3 \mathrm{~T} 3 \mathrm{~L} 1$ adipocytes, ${ }^{36}$ with a similar effect of atorvastatin in mouse white adipose tissue, thereby causing insulin resistance. ${ }^{28}$ Statins reduce the intermediate pathway of mevalonic acid beyond cholesterol. Inhibition of the mevalonate pathway reduces insulin-stimulated lipogenesis in adipose tissue instead of glucose uptake or inhibition of lipolysis, signs indicative of the development of insulin resistance. Reduction of statin prenylation inhibits insulin signaling in adipose tissue and reduces autonomic lipocyte lipogenesis. In rats, the statin-induced decrease in isoprenoids required for protein propylation resulted in activation of inflammatory NLRP3 / caspase-1 and interleukin-1 $\beta$ (IL-1 $\beta$ ). IL-1 $\beta$ was the major mediator of statin-induced insulin resistance in adipocytes, which eventually attenuated lipogenesis. IL-1 $\beta$ alone reduces insulin-stimulated lipogenesis in rodent and human adipocytes. ${ }^{37}$ In addition, statins appear to reduce the secretion of high molecular weight oligomers of adiponectin by a mechanism that reduces insulin sensitivity. Interestingly, statins also affect the process of differentiation of pre-adipocytes into adipocytes. The mechanism underlying this effect is most likely the lack of secretion of insulin sensitizing hormones. This has been shown to be caused by decreased expression of PPARY (peroxisome proliferator - activated receptor Y) and C / EBP (CCAAT / enhancer-binding protein) transcription factors. ${ }^{26,38}$ 


\section{ii. Skeletal muscle and statins}

Skeletal muscle consumes most of the glucose entering the circulation ( $75 \%$ of glucose is available throughout the body) and any disturbance of glucose uptake by this tissue can lead to the development of new onset type 2 diabetes..$^{55}$ GLUT-4, which mediates glucose transport in skeletal muscle cells, is a key factor in controlling blood glucose. As mentioned above, insulin binding to the INSR causes Akt activation and displacement of GLUT-4-containing vesicles in the plasma membrane facilitating glucose transport. ${ }^{55}$

Although the mechanism of statin-induced new onset type 2 diabetes is not fully understood, there are both in vivo and in vitro studies that shed some light on this phenomenon in skeletal muscle. Some of the mechanisms that have been previously described are statin-mediated inhibition of insulin stimulated glucose uptake, impairment of intracellular signaling of the INSR and thereby of the Akt/mTOR pathway, or an excess of FFA accumulation in skeletal muscle as a consequence of HMG-CoA reductase inhibition. ${ }^{40}$

Simvastatin reduces GLUT-4 expression in L6 tubules, increasing the risk of new onset type 2 diabetes. Assessment of the mechanism of simvastatin-associated impairment of glucose transport into myotubes suggests that impaired intracellular signaling of the INSR pathway also plays an important role. Simvastatin treatment increase the risk of new onset diabetes by reducing both insulin secretion, and insulin sensitivity, suppressing glucose uptake by inhibiting critical steps in the IR / IRS-1 / AKT signaling sequence and inhibiting GLUT4 function and normal regulation of glucose synthesis to insulin resistance, whereas pravastatin, which has different physicochemical properties, does not inhibit insulin-induced glucose uptake, resulting in protection from developing new onset type 2 diabetes. ${ }^{56}$ This insufficient GLUT-4 shift is most likely caused by attenuated Akt-mediated phosphorylation of GSK3 $\beta$. In addition, simvastatin treatment induces higher plasma glucose levels in mice despite increased plasma insulin concentrations, a consequence of insulin resistance. ${ }^{39}$ Alternatively, it has been suggested that simvastatin may induce insulin resistance through a new fatty acidbased mechanism, regardless of the effects of lowering cholesterol. ${ }^{40}$ Simvastatin has been shown to inhibit the accumulation of HMG CoA reductase, which can lead to the accumulation of acetyl CoA, a precursor to the synthesis of fatty acids that can promote intracellular fatty acid accumulation. The resulting excess of FFA accumulation in skeletal muscle can inhibit glucose uptake by reducing GLUT-4 shift. ${ }^{40-57}$

\section{iii. The liver in statin treatment}

The liver plays a central role in glucose homeostasis and is extremely sensitive to insulin. In fact, insulin regulates many hepatic metabolic pathways ranging from glucose production to lipid synthesis. A decrease in hepatic insulin sensitivity is rapidly reflected in glucose and triglyceride homeostasis levels, with worsening glycemic control. ${ }^{41}$ Statin therapy is associated with a small increment in fasting blood glucose levels. Atorvastatin affects glucose utilization in hepatocytes by suppressing GLUT2 and GCK expression, which may be due in part to PXR activation. ${ }^{41}$

Statins have been shown to stimulate endogenous glucose production by activating phosphoenylpyruvate carboxykinase (PEPCK) and glucose-6-phosphatase (G6Pase), ${ }^{42}$ the major glycogenic enzymes in human cells. Increased hepatic gluconeogenesis contributes to hyperglycemia, a characteristic of insulin resistance and T2DM.

Regarding FFAs, an excess of FFA accumulation in liver cells may also contribute to the development of T2DM. Interestingly, treatment with atorvastatin and rosuvastatin upregulates the expression of thyroid hormoneresponsive spot 14 (THRSP) protein, a small protein primarily expressed in lipid-producing tissues, such as those found in the liver. THRSP has been implicated as a regulator of lipogenic processes by controlling the expression of lipogenic genes, such as fatty acid synthase (FASN), ATP citrate lyase (ACLY) SREBP and ChREBP or their activity. ${ }^{57,58}$

\section{Statin therapy and Micro-RNAs (miRs)}

MicroRNAs (miRs) are small (22 nucleotides) unencoded RNA regulators that act as post-transcriptional regulators of gene expression. ${ }^{43}$ MiRs have been shown to be involved in many biological processes such as insulin expression, skeletal muscle adaptation to elevated glucose, insulin sensitivity, and glucose-stimulated insulin secretion (GSIS). Statin therapy affects the expression of several miRs, which play a central role in regulating lipid and glucose metabolism ${ }^{44}$ and are associated with the development of diabetes.

Simvastatin and atorvastatin induce expression of miR-33a in the liver suggesting an association between decreased insulin secretion and, ultimately, statin-induced increase in the incidence of type 2 diabetes. Incubation of hepatocytes with atorvastatin, simvastatin or pravastatin regulate the expression of the essential gluconeogenic enzymes PEPCK and G6Pase. Statin-mediated effects include miR-183/96/182-mediated regulation of transcription factor 7-type 2 (TCF7L2), which regulates hepatic and 
peripheral glucose metabolism and whole-body glycemic control. ${ }^{32,45}$

These results suggest that patients on long-term statin treatment may have a persistently increased miR complex expression leading to prolonged activation of the glycogenetic pathway. The effect ofpitavastatin, on macrophage miRNAs in the presence or absence of oxidized LDL-C suggests that pitavastatin may regulate miRNA differentially in the presence of oxLDL with a net effect on cholesterol homeostasis. ${ }^{46}$

\section{CONCLUSIONS}

Statins have revolutionized our way of looking at atherosclerotic cardiovascular disease. However, they have also been shown to have side effects, which although not very common, can be serious. The risk of new onset diabetes attributed to statins is a deterrent for patients, and even for medical personal. It should be stressed that the beneficial properties of statins by far outweigh any risk associated the drug. Some statins are more prone to side effects e.g., simvastatin, rosuvastatin and atorvastatin compared to others e.g., pravastatin and pitavastatin. Those on statins should be followed regularly, especially during the first months after starting therapy for possible new onset diabetes.

\section{Conflict of interest}

The are no conflicts of interest

\title{
ПЕРІАНЧН
}

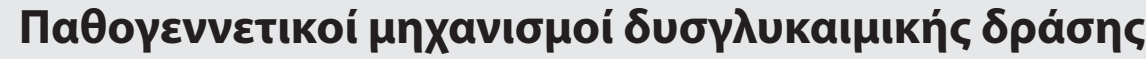

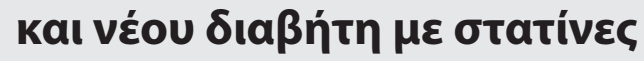

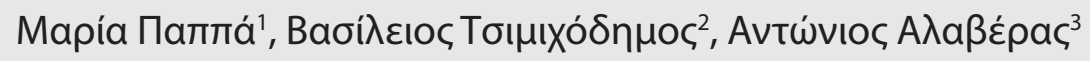

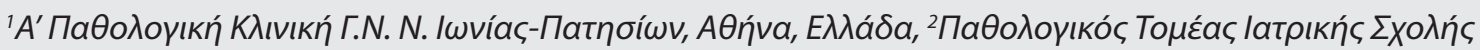

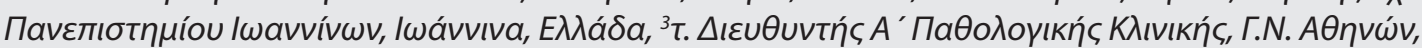

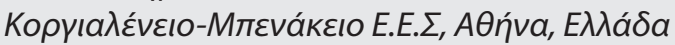

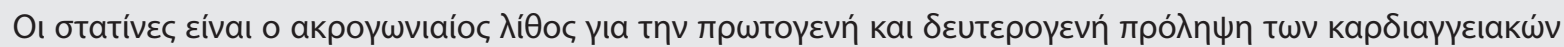

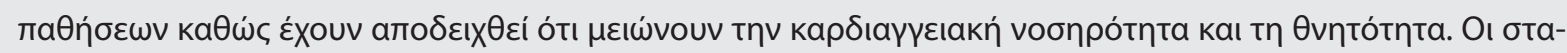

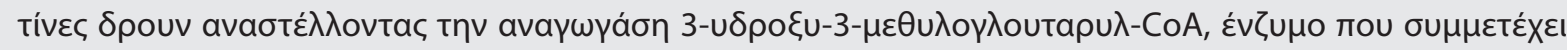

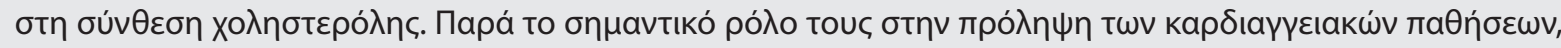

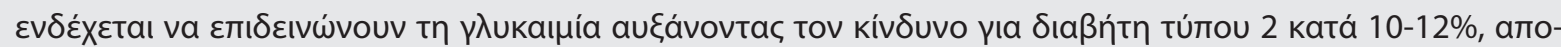

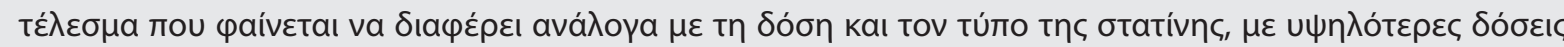

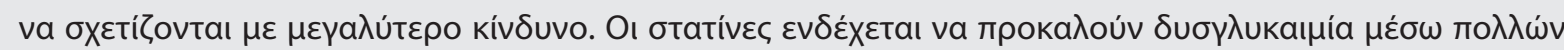

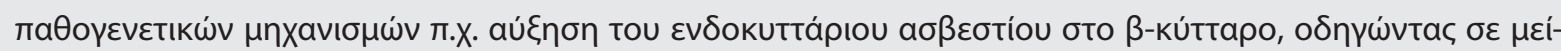

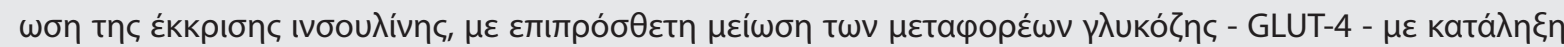

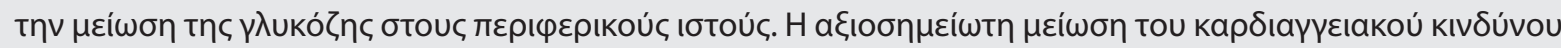

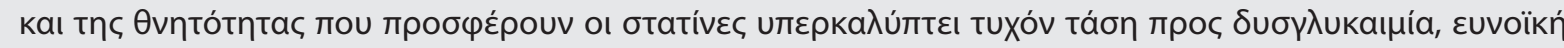

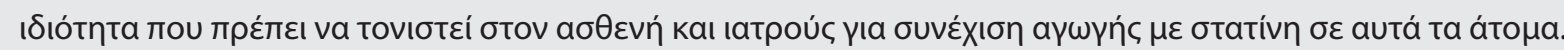

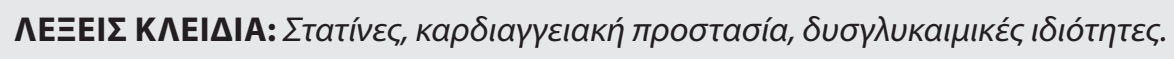

\section{REFERENCES}

1. The Emerging Risk Factors Collaboration, Sarwar $N$, Gao P, Kondapally Seshasai SR, Gobin R, Kaptoge S, Di Angelantonio $\mathrm{E}$, et al. Diabetes mellitus, fasting blood glucose concentration, and risk of vascular disease: $\mathrm{A}$ collaborative meta-analysis of 102 prospective studies. Lancet. 2010 Jun;375(9733):2215-22.

2. Lindahl B, Toss H, Siegbahn A, Venge P, Wallentin L. Markers of myocardial damage and inflammation in relation to long-term mortality in unstable coronary artery disease. FRISC Study Group. Fragmingham during Instability in Coronary Artery Disease. N Engl J Med. 2000 Oct;343(16):1139-47.

3. Baigent C, Keech A, Kearney PM, Blackwell L, Buck G, Pollicino $C$, et al. Efficacy and safety of cholesterol-lowering treatment: prospective meta-analysis of data from 90,056 participants in 14 randomized trials of statins. Lancet. 2005 Oct;366(9493):1267-78. 
4. Cholesterol Treatment Trialists' Collaboration, Baigent C, Blackwell L, Emberson J, Holland LE, Reith C, Bhala $\mathrm{N}$, et al. Efficacy and safety of more intensive lowering of LDL cholesterol: A meta-analysis of data from 170,000 participants in 26 randomized trials. Lancet. 2010 Nov;376(9753):1670-81.

5. Jain MK, Ridker PM. Anti-inflammatory effects of statins: Clinical evidence and basic mechanisms. Nat Rev Drug Discov. 2005 Dec;4(12):977-87.

6. Collins R, Reith C, Emberson J, Armitage J, Baigent C, Blackwell $L$, et al. Interpretation of the evidence for the efficacy and safety of statin therapy. Lancet. 2016 Nov;388(10059):2532-61.

7. Ganga HV, Slim HB, Thompson PD. A systematic review of statin-induced muscle problems in clinical trials Am Heart J. 2014 Jul;168(1):6-15.

8. Sattar N, Preiss D, Murray HM, Welsh P, Buckley BM, de Craen AJM, et al. Statins and risk of incident diabetes: $A$ collaborative meta-analysis of randomised statin trials. Lancet. 2010 Feb;375(9716):735-42.

9. Casula M, Mozzanica F, Scotti, L, Tragni E, Pirillo A, Corrao $G$, et al. Statin use and risk of new-onset diabetes: A meta-analysis of observational studies. Nutr Metab Cardiovasc Dis. 2017 May;27(5):396-406.

10. Ridker, PM, Pradhan A, MacFadyen JG, Libby P, Glynn RJ. Cardiovascular benefits and diabetes risks of statin therapy in primary prevention: An analysis from the JUPITER trial. Lancet. 2012 Aug;380(9841):565-71.

11. Agarwala A, Kulkarni S, MaddoxT. The association of statin therapy with incident diabetes: Evidence, mechanisms, and recommendations. Curr Cardiol Rep. 2018 May; 19;20(7):50.

12. Mills EJ, Wu P, Chong G, Ghement I, Singh S, Akl EA, et al. $M$ Efficacy and safety of statin treatment for cardiovascular disease: A network meta-analysis of 170,255 trials 70,255 patients from 76 randomized trials. QJM. 2011 Feb;104(2):109-24.

13. Carter AA, Gomes T. Risk of incident diabetes among patients treated with statins: Population based study. BMJ [Internet]. 2013 May;346:f2610. Available from: https:// www.ncbi.nlm.nih.gov/pmc/articles/PMC3898149/

14. Zaharan NL, William D, Bennett K. Statins and risk of treated incident diabetes in a primary care population. — Br J Clin Pharmacol. 2013 Apr;75(4):1118-24.

15. Cederberg H, Stancakova A, Yaluri N, Modi S, Kuusisto J, Laakso M. Increased risk of diabetes with statin treatment is associated with impaired insulin sensitivity and insulin secretion: A 6 year follow-up study of the METSIM cohort. Diabetologia. 2015 May;58(5):1109-17.

16. Yamakawa T, Takano T, Tanaka S, Kadonosono K, Terauchi Y. Influence of pitavastatin on glucose tolerance in patients with type 2 diabetes mellitus. J Atheroscler Thromb. 2008 Oct;15(5):269-75.

17. Park ZH, Juska A, Dyakov D, Patel RV. Statin-associated incident diabetes: A literature review. Consult Pharm. 2014;29(5):317-34.

18. Neil HAW, DeMicco DA, Luo D, Betteridge DJ, Colhoun $\mathrm{H}$, Durrington PN, et al. Analysis of efficacy and safety in patients aged $65-75$ years at randomization: Collaborative Atorvastatin Diabetes Study (CARDS). Diabetes Care 2006 Nov;29(11):2378-84.

19. Angelidi AM, Stambolliu E, Adamopoulou KI, Kousoulis
A. Is atorvastatin associated with new onset diabetes or deterioration of glycemic control? Systematic Review using data from 1.9 million patients. Int J Endocrinol [Internet]. 2018 Oct;8380192. Available from: https:// pubmed.ncbi.nlm.nih.gov/30425742/

20. Waters DD, Ho JE, DeMicco DA, Breazna A, Arsenault BJ, Wun C-C, et al. Predictors of new-onset diabetes in patients treated with atorvastatin: Results from 3 large randomized clinical trials. J Am Coll Cardiol. 2011 Apr;57(14):1535-45.

21. Preiss D, Seshasai SR, Welsh P, Murphy SA, Ho JE, Waters $D D$, et al. Risk of incident diabetes with intensive-dose compared with moderate-dose statin therapy: A metaanalysis. JAMA. 2011 Jun;305(24):2556-64.

22. Rajpathak SN, Kumbhani DJ, Crandall J, Barzilai N, Alderman MA, Ridker PM. Statin therapy and risk of developing type 2 diabetes: A meta-analysis. Diabetes Care. 2009 Oct;32(10):1924-9.

23. Cholesterol Treatment Trialists' (CTT) Collaborators; Kearney PM, Blackwell L, Collins R, Keech A, Simes J, Peto R, et al. Efficacy of cholesterol-lowering therapy in 18,686 people with diabetes in 14 randomized trials of statins: 2 A meta-analysis. Lancet. 2008 Jan;371(9607):117-25.

24. Samuel VT, Shulman GI. The pathogenesis of insulin resistance: Integrating signaling pathways and substrate flux. J Clin Invest. 2016 Jan;126(1):12-22.

25. Brault M, Ray J, Gomez VH, Mantzoros CS, Daskalopoulou SS. Statin treatment and new-onset diabetes: A review of proposed mechanisms. Metabolism. 2014 Jun;63(6):735-45.

26. Katsiki N, Rizzo M, Mikhailidis DP, Mantzoros CS. Newonset diabetes and statins: Throw the bath water out, but please keep the baby. Editorial. Metabolism. 2015 April;64(4):471-5.

27. Nakata M, Nagasaka S, Kusaka I, Matsuoka H, Ishibashi $S$, Yada Y. Effects of statins on the adipocyte maturation and expression of glucose transporter 4 (SLC2A4): Implications in glycaemic control. Diabetologia. 2006 Aug;49(8):1881-92.

28. Zhao W, Zhao SP. Different effects of statins on induction of diabetes mellitus: An experimental study. Drug Des. Dev Ther. 2015 Nov;9:6211-23.

29. Galicia-Garcia U, Jebari S, Sebal AL, Uribe KB. Uribe KB, Siddiqi $H$, et al. Statin treatment-induced development of type 2 diabetes: From clinical evidence to mechanistic insights. J Mol Sci. 2020 Jul;21(13):4725.

30. Kruit JK, Brunham LR, Verchere CB, Hayden MR. HDL and LDL cholesterol significantly influence beta-cell function in type 2 diabetes mellitus. Curr Opin Lipidol. 2010 Jun;21(3):178-85.

31. Khan S, Yan-Do R, Duong E, Wu X, Bautista A, Cheley S, et al. Autocrine activation of $\mathrm{P} 2 \mathrm{Y} 1$ receptors couples $\mathrm{Ca}$ $(2+)$ influx to $\mathrm{Ca}(2+)$ release in human pancreatic beta cells. Diabetologia. 2014 Dec;57(12):2535-45.

32. Shi MY, Bang IH, Han CY, Lee DH, Park BH, Bae EJ. Statin suppresses sirtuin 6 through miR-495, increasing FoxO1dependent hepatic gluconeogenesis. Theranostics. 2020 Sep;10(25):11416-27.

33. Barkas F, Elisaf M, Liberopoulos E, Liamis G, Ntzani E, Rizos EC. Atherogenic dyslipidemia increases the risk of incident diabetes in statin-treated patients with impaired 
fasting glucose or obesity. J Cardiol. 2019 Sep;(3);74:290-5.

34. Curry L, Almukhtar H, Alahmed J, Roberts R, Smith PA. Simvastatin inhibits L-Type Ca2+-Channel Activity through impairment of mitochondrial function. Toxicol Sci. 2019 Jun;169(2):543-52.

35. Mollazadeh H, Tavana E, Fanni G, Bo S, Banach M, Pirro $M$, et al. Effects of statins on mitochondrial pathways. J Cachexia Sarcopenia Muscle. 2021 Jan;1-14.

36. Dludla PV, Nyambuya TM, Orlando P, Silvestri S, Mxinwa V, Mokgalaboni K, et al. The impact of coenzyme Q10 on metabolic and cardiovascular disease profiles in diabetic patients: A systematic review and meta-analysis of randomized controlled trials. Endocrinol Diabetes Metab [Internet]. 2020 Apr;3(2):e00118. Available from: https:// www.ncbi.nlm.nih.gov/pmc/articles/PMC7170462/

37. Satoh K, Itagaki M, Tokumitsu Y, Ichihara. Effects of atorvastatin and pravastatin on signal transduction related to glucose uptake in 3T3L1 adipocytes. J Pharmacol Sci. 2008 May; 107(1):80-9.

38. Henriksbo BD, Tamrakar AK, Xu J, Duggan BM, Cavallari JF, Phulka J, et al. Statins promote interleukin-1b-dependent adipocyte insulin resistance through lower prenylation, not cholesterol. Diabetes. 2019 Jul;68(7):1441-8.

39. Moutzouri E, Liberopoulos E, Mikhailidis DP, Kostapanos MS, Kei AA, Milionis $H$, et al. Comparison of the effects of simvastatin vs. rosuvastatin vs. simvastatinezetimibe on parameters of insulin resistance. Int J Clin Pract. 2011 Nov;65(11):1141-8.

40. Sanvee GM, Panajatovic MV, Bouitbir J, Krahenbuhl S. Mechanisms of insulin resistance by simvastatin in $\mathrm{C} 2 \mathrm{C} 12$ myotubes and in mouse skeletal muscle. Biochem Pharm. 2019 Jun;164:23-33.

41. Kain V, Kapadia, B, Misra P, Saxena U. Simvastatin may induce insulin resistance through a novel fatty acid mediated cholesterol independent mechanism. Sci Rep. 2015 Sep;5:3-9.

42. Ling Z, Shu N, Xu P, Wang F, Zhong, Sun Z, et al. Involvement of pregnane $X$ receptor in the impaired glucose utilization induced by atorvastatin in hepatocytes. Biochem Pharm. 2016 Jan;100:98-111

43. Gotoh S, Negishi M. Statin-activated nuclear receptor PXR promotes SGK2 dephosphorylation by scaffolding PP2C to induce hepatic gluconeogenesis. Sci Rep. 2015 Sep;5:14076.

44. FilipowiczW, Bhattacharyy SN, Sonenberg N. Mechanisms of post-transcriptional regulation by microRNAs: Are the answers in sight? Nat Rev Genet. 2008 Feb;9:102-14.

45. Fernandez-Hernando C, Ramirez CM, Goedeke L, Suarez Y. MicroRNAs in metabolic disease. Arterioscler Thromb Vasc Biol. 2013 Feb;33(2):178-85.
46. Marquart TJ, Allen RM, Chen MR, Dorn GW, II Matkovich SJ, Baldan A. Statins stimulate hepatic glucose production via the miR-183/96/182 Cluster. BioRxiv [Internet]. 2019 Aug; Available from: https://www.biorxiv.org/ content/10.1101/726695v1

47. Zhang H, Lamon BD, Moran G, Sun T, Gotto AM Jr, Hajjar DP. Pitavastatin differentially modulates microRNAassociated cholesterol transport proteins in macrophages. PLoS ONE [Internet]. 2016 Jul [cited YEAR MONTH DAY]; e0159130. Available from: https://journals.plos.org/plosone/article?id=10.1371/journal.pone.0159130

48. Kostapanos MS, Liamis GL, Milionis HJ, Elisaf MS. Do statins beneficially or adversely affect glucose homeostasis? Curr Vasc Pharm. 2010 Sep;8(5):612-31.

49. Lizcano JM, Alessi DR. The insulin signalling pathway. Curr Biol. 2002 Apr;12(7):R236-8.

50. Copps KD, White MF. Regulation of insulin sensitivity by serine/threonine phosphorylation of insulin receptor substrate proteins IRS1 and IRS2. Diabetologia. 2012 Oct; 55(10):2565-82.

51. Ho CK, Sriram G, Dipple KM. Insulin sensitivity predictions in individuals with obesity and type II diabetes mellitus using mathematical model of the insulin signal transduction pathway. Mol Genet Metab. 2016 Nov;119(3):288-92.

52. Huang S, Czech MP. The GLUT4 glucose transporter. Cell Metab. 2007 Apr;5(4):237-52.

53. Moraes-Vieira PM, Saghatelian A, Kahn BB. GLUT4 expression in adipocytes regulates de novo lipogenesis and levels of a novel class of lipids with antidiabetic and antiinflammatory effects. Diabetes. 2016 Jul;65(7):1808-15.

54. Taniguchi CM, Emanuelli B, Kahn CR. Critical nodes in signalling pathways: Insights into insulin action. Nat Rev Mol Cell Biol. 2006 Feb;7(2):85-96.

55. Carnagarin R, Dharmarajan AM, Dass CR. Molecular aspects of glucose homeostasis in skeletal muscle-A focus on the molecular mechanisms of insulin resistance. Mol Cell Endocrinol. 2015;417:52-62.

56. Yaluri N, Modi S, Kokkola T. Simvastatin induces insulin resistance in $\mathrm{L} 6$ skeletal muscle myotubes by suppressing insulin signaling, GLUT4 expression and GSK-3beta phosphorylation. Biochem Biophys Res Commun. 2016 Nov;480(2):194-200.

57. Zhu Q, Anderson GW, Mucha GT, Parks EJ, Metkowski $\mathrm{JK}$, Mariash CN. The Spot 14 protein is required for de novo lipid synthesis in the lactating mammary gland. Endocrinology. 2005 Aug;146(8):3343-50.

58. Colbert CL, Kim CW, Moon YA, Henry L, Palnitkar M, McKean WB, et al. Crystal structure of Spot 14, a modulator of fatty acid synthesis. Proc Natl Acad Sci. USA 2010 Nov;107(44):18820-5. 